\title{
Bofutsushosan, a Japanese herbal (Kampo) medicine, attenuates progression of nonalcoholic steatohepatitis in mice
}

\author{
Masafumi Ono - Mitsunari Ogasawara - Akira Hirose - Sachiko Mogami • Nobuhiro Ootake • \\ Kosuke Aritake • Takuma Higuchi - Nobuto Okamoto • Shuji Sakamoto • Masahiro Yamamoto • \\ Yoshihiro Urade · Toshiji Saibara · Jude A. Oben
}

Received: 17 October 2012/ Accepted: 13 June 2013/Published online: 26 June 2013

(C) The Author(s) 2013. This article is published with open access at Springerlink.com

\begin{abstract}
Background Obesity-induced liver disease (nonalcoholic fatty liver disease, NAFLD) is now the commonest cause of chronic liver disease in affluent nations. There are presently no proven treatments for NAFLD or its more severe stage, nonalcoholic steatohepatitis (NASH). Bofutsushosan (BTS), a Japanese herbal (Kampo) medicine, long used as an anti-obesity medicine in Japan and other Asian countries, has been shown to reduce body weight and improve insulin resistance (IR) and hepatic steatosis. The precise mechanism of action of BTS, however, remains
\end{abstract}

Electronic supplementary material The online version of this article (doi:10.1007/s00535-013-0852-8) contains supplementary material, which is available to authorized users.

M. Ono $(\bowtie) \cdot$ M. Ogasawara · A. Hirose · N. Okamoto ·

T. Saibara

Department of Gastroenterology and Hepatology, Kochi Medical

School, Kohasu, Nankoku, Kochi 783-8505, Japan

e-mail: onom@kochi-u.ac.jp

S. Mogami $\cdot$ N. Ootake $\cdot$ M. Yamamoto

Tsumura Research Laboratories, Tsumura \& Co., Ibaraki, Japan

K. Aritake · Y. Urade

Department of Molecular Behavioral Biology, Osaka Bioscience

Institute, Osaka, Japan

T. Higuchi $\cdot$ S. Sakamoto

Laboratory of Molecular Biology, Science Research Center,

Kochi Medical School, Kochi, Japan

J. A. Oben

Institute for Liver and Digestive Health, University College

London, London, UK

J. A. Oben

Department of Gastroenterology and Hepatology,

Guy's and St. Thomas' Hospital, London, UK unclear. To evaluate the ability of BTS to prevent the development of NASH, and determine the mediators and pathways involved.

Methods C57BL/6 mice were injected intra-peritoneally with gold-thioglucose and fed a high-fat diet (HF) or HF diet admixed with either 2 or $5 \%$ BTS for 12 weeks. The effectiveness of BTS in attenuating features of NASH and the mechanisms through which BTS attenuated NASH were then assayed through an assessment of the anthropometric, radiological, biochemical and histological parameters.

Results BTS attenuated the progression of NASH through induction of adiponectin and its receptors along with an induction of PPAR- $\alpha$ and PPAR- $\gamma$, decreased expression of SREBP-1c, increased hepatic fatty acid oxidation and increased hepatic export of triglycerides. BTS moreover, reduced IR through phosphorylation of the protein kinase, Akt.

Conclusions BTS through induction of adiponectin signaling and Akt attenuated development of NASH. Identification of the active entity in BTS should allow development of novel treatments for NASH.

Keywords NAFLD - Adiponectin · Bofutsushosan · Kampo medicine

\begin{tabular}{ll}
\multicolumn{2}{l}{ Abbreviations } \\
NAFLD & Nonalcoholic fatty liver disease \\
NASH & Nonalcoholic steatohepatitis \\
GTG & Gold-thioglucose \\
HF & High-fat-diet \\
BTS & Bofutsushosan \\
IR & Insulin resistance \\
IGT & Impaired glucose tolerance \\
GTT & Glucose tolerance test
\end{tabular}


ITT Insulin tolerance test

QUICKI Quantitative insulin sensitivity check index

MCAD Medium-chain acyl-CoA dehydrogenase

TIMP Tissue inhibitor of metalloproteinases

TGF Transforming growth factor

MTP Microsomal triglyceride transfer protein

AMPK AMP-activated protein kinase

\section{Introduction}

Obesity-induced liver disease (nonalcoholic fatty liver disease, NAFLD), is now the commonest cause of chronic liver disease in affluent nations. The disease comprises obesity and insulin resistance (IR), with a consequent histopathological spectrum of hepatosteatosis, steatohepatitis (nonalcoholic steatohepatitis, NASH), cirrhosis and possible hepatocellular cancer [1-3]. There are presently no proven treatments for NAFLD or its more severe stage NASH. Bofutsushosan (BTS), a Japanese herbal (Kampo) medicine, long used as an anti-obesity medicine in Japan and other Asian countries [4] has recently been shown in obese Japanese women, to reduce body weight and improve IR [4]. In addition, BTS in experimental animals prevented adipogenesis [5], reduced weight, suppressed visceral and subcutaneous fat accumulation and in parallel decreased plasma glucose, triglycerides (TG), insulin, tumor necrosis factor-alpha [6], and hepatic steatosis induced by high-fat diet feeding [7, 8]. The mechanism of action of BTS however, is not known. Our aim here was to evaluate the ability of BTS to prevent the development of NASH in a recently described murine model involving administration of gold-thioglucose (GTG) and high-fat feeding to induce NASH [9], and to determine the mediators and pathways involved.

\section{Materials and methods}

Animal preparation

All procedures conformed to our institutions' guidelines for the care and use of animals in Kochi Medical School. Fourweek-old male C57BL/6 mice were purchased from CLEA Japan Inc. All animals were housed for 12 weeks on a $12 \mathrm{~h}$ light/12 h dark cycle, with food and water freely available. Mice were fed high-fat-diet (HF, 640 kcal/100 g, F2HFD2, Oriental Yeast, Tokyo, Japan) or HF admixed with 2 or $5 \%$ BTS (TJ-62, Tsumura \& Co., Tokyo, Japan). All groups were fed standard chow (SC) for the first week and then continued on their respective group diets for remainder of the protocol.

Three experimental groups were studied: (1) intra-peritoneal administered GTG (2 mg/g of body weight, SigmaAldrich, St. Louis, MO, USA) and then SC for 1 week followed by HF diet for 11 weeks (GTG + HF) [9]; (2 and 3) intra-peritoneal GTG, SC for 1 week followed by HF diet admixed with either $2 \%$ BTS or $5 \%$ BTS for further 11 weeks ( $2 \%$ BTS or $5 \%$ BTS). At the end of the treatment period, all animals were fasted overnight, anesthetized with pentobarbital sodium intraperitoneally $(25-50 \mathrm{mg} / \mathrm{kg}$ of body weight, Nembutal; Abbott Laboratories, Abbott Park, IL, USA). Blood and liver samples were harvested. Livers were fixed in $10 \%$ formalin, or snap frozen in liquid nitrogen and stored at $-80^{\circ} \mathrm{C}$, for later analyses.

CT scan analysis for body fat composition

The extent of adiposity in each experimental group was assayed by CT scanning (La Theta, ALOKA, Tokyo, Japan) under isoflurane $(2 \% \mathrm{v} / \mathrm{v})$ anesthesia as described previously [9]. Animal were scanned at 2-mm intervals from the diaphragm to the pelvis, and visceral fat and subcutaneous fat volumes quantified with La Theta software (version 1.00) [9-11].

\section{Histopathological examination}

Five-micrometer sections of formalin-fixed/paraffinembedded livers were processed for haematoxylin and eosin (H\&E). Oil Red-O staining of intracellular neutral lipids was performed according to the manufacturer's instructions (Sigma-Aldrich, St. Louis, MO, USA). For estimation of extent of hepatic steatosis, the areas of digital photomicrographs were quantified with a computerized image analysis system (macintosh MacSCOPE version $2.591)$ as described previously $[9,12]$. Degree of oxidative stress was determined by staining and quantification with anti-8-hydroxy-2'-deoxyguanosine (8-OHdG) and anti-4hydroxy-2-nonenal (4-HNE) as previously described [9, $12,13]$.

Glucose tolerance test (GTT), insulin tolerance test (ITT) and QUICKI

At 12 weeks, a glucose tolerance test (GTT) $(n=6)$ and an insulin tolerance test (ITT) $(n=6)$ were performed. For GTT, mice were fasted for $18 \mathrm{~h}$, and then intra-peritoneally loaded with $20 \%$ glucose at a dose of $1.0 \mathrm{~g} / \mathrm{kg}$ body weight. For ITT, mice were fasted for $6 \mathrm{~h}$, and then intraperitoneally challenged with human insulin at $1.0 \mathrm{U} / \mathrm{kg}$ body weight $[14,15]$. With both GTT and ITT blood 
samples from the orbital sinus were taken at times 0,30 , 45, 60 and $120 \mathrm{~min}$ and plasma glucose concentrations measured using an automatic blood glucose meter (Glutest; Sanwa Kagaku Kenkyusho Co., Ltd., Nagoya, Japan). Plasma insulin level was measured by Ultrasensitive Mouse Insulin ELISA kit (Mercodia AB, Uppsala, Sweden) according to the manufacture's protocol. The quantitative insulin sensitivity check index (QUICKI), as a measure of IR, was calculated from the fasting insulin and glucose levels.

Measurement of plasma adiponectin

Plasma adiponectin levels were measured by Mouse Adiponectin/Acrp30 (R\&D Systems, Minneapolis, MN, USA) according to the manufacture's instructions.

Laboratory evaluation

Asparate aminotransferase (AST), alanine aminotransferase (ALT) and TG were measured by an autoanalyzer (BM6010; JEOL Ltd., Tokyo, Japan).

Real-time RT-PCR for quantitative assessment of mRNA expression

Total RNA was extracted using trizol reagent (Life Technologies, Grand Island, NY, USA) according to the manufacture's protocol. RNA extracts were reverse-transcribed with random hexamers and avian myeloblastosis virus reverse transcriptase using a commercial kit (Takara, Kyoto, Japan). Real time RT-PCR were performed for quantitative assessment of mRNA expression on an ABI Prism 7000 Sequence Detection system (Applied Biosystems, Foster City, CA, USA) according to the manufacturer's protocol. Probes and primers for TNF- $\alpha$, PPAR- $\alpha$, PPAR- $\gamma$, MTP, DGAT2, Cyp2E1, adiponectin receptor 1/receptor 2 (AdipoR1/R2) were all purchased from Applied Biosystems. Relative expression of target gene mRNA was normalized to the amount of GAPDH mRNA.

Western blot analysis

For in vivo analysis of phosphorylated Akt and total Akt, mice were fasted for $18 \mathrm{~h}$, injected intra-peritoneally with human insulin (10 U/kg) or control, and sacrificed $4 \mathrm{~min}$ later. Livers were snap frozen in liquid nitrogen. Liver total protein was analyzed by western blot with a polyclonal antibody to phosphorylated Akt/Akt and phosphorylated AMPK/AMPK (Cell Signaling Technology, Inc., Danvers, MA, USA) as described [16]. For analysis of SREBP-1c, mice were fasted for $18 \mathrm{~h}$, sacrificed, and livers dissected and homogenized to prepare cell nuclear extracts which were then analyzed by western blotting with anti-SREBP1c antibody (Santa Cruz Biotechnology, Inc., California, CA, USA) as described [17]. For analysis of 4-HNE, liver total protein was analyzed by western blot with a monoclonal anti-4-HNE antibody (Japan Institute for the Control of Aging, Shizuoka, Japan).

Statistics

Data are shown as mean $\pm \mathrm{SD}$. A univariate analysis was conducted with the Mann-Whitney $U$ test to determine significance between groups. Qualitative data were compared using Fisher's exact test. Statistical significance was accepted at $p<0.05$. All analyses were performed using Stat View software (SAS Institute, Cary, NC, USA).

\section{Results}

BTS treatment reduces GTG + HF induced obesity and steatohepatitis

Mice administered GTG and then fed a HF diet $(\mathrm{GTG}+\mathrm{HF})$, had a comprehensive histological and dysmetabolic phenotype resembling human NASH as reported recently [9]. To then evaluate the effectiveness of BTS in the GTG + HF model, we studied the anthropometric, radiological, biochemical and histological parameters as detailed above in the presence or absence of BTS 2 or $5 \%$ admixed with the HF component of the GTG $+\mathrm{HF}$. Administration of BTS attenuated weight gain in a dose dependent manner (Fig. 1a). Unexpectedly, GTG + HF induced increase in the volume of the visceral and subcutaneous fat were not markedly attenuated by treatment with BTS (Fig. 1b). However, hepatic steatosis on H\&E or Oil Red-O staining in 12 weeks was attenuated in a dose dependent manner by BTS treatment (Figs. 2a, 3a). Additionally, BTS treatment also attenuated GTG + HF induced hepatomegaly in a dose-dependent manner (Fig. 2b).

GTG + HF mice livers showed steatohepatitis with marked steatosis and inflammation, hepatocyte ballooning and Mallory-Denk bodies as described previously (Fig. 3a) [9]. BTS treatment attenuated hepatic steatosis and hepatic inflammation (Fig. 3a), and inhibited hepatocyte ballooning and Mallory-Denk bodies. In parallel, oxidative stress makers, 8-OHdG (Fig. 3b) and 4-HNE (supplemental figure), were remarkably reduced by BTS, as was the expression of TNF- $\alpha$ (Fig. 3b). BTS treatment moreover, attenuated GTG + HF induced elevation of transaminases (Table 1). 


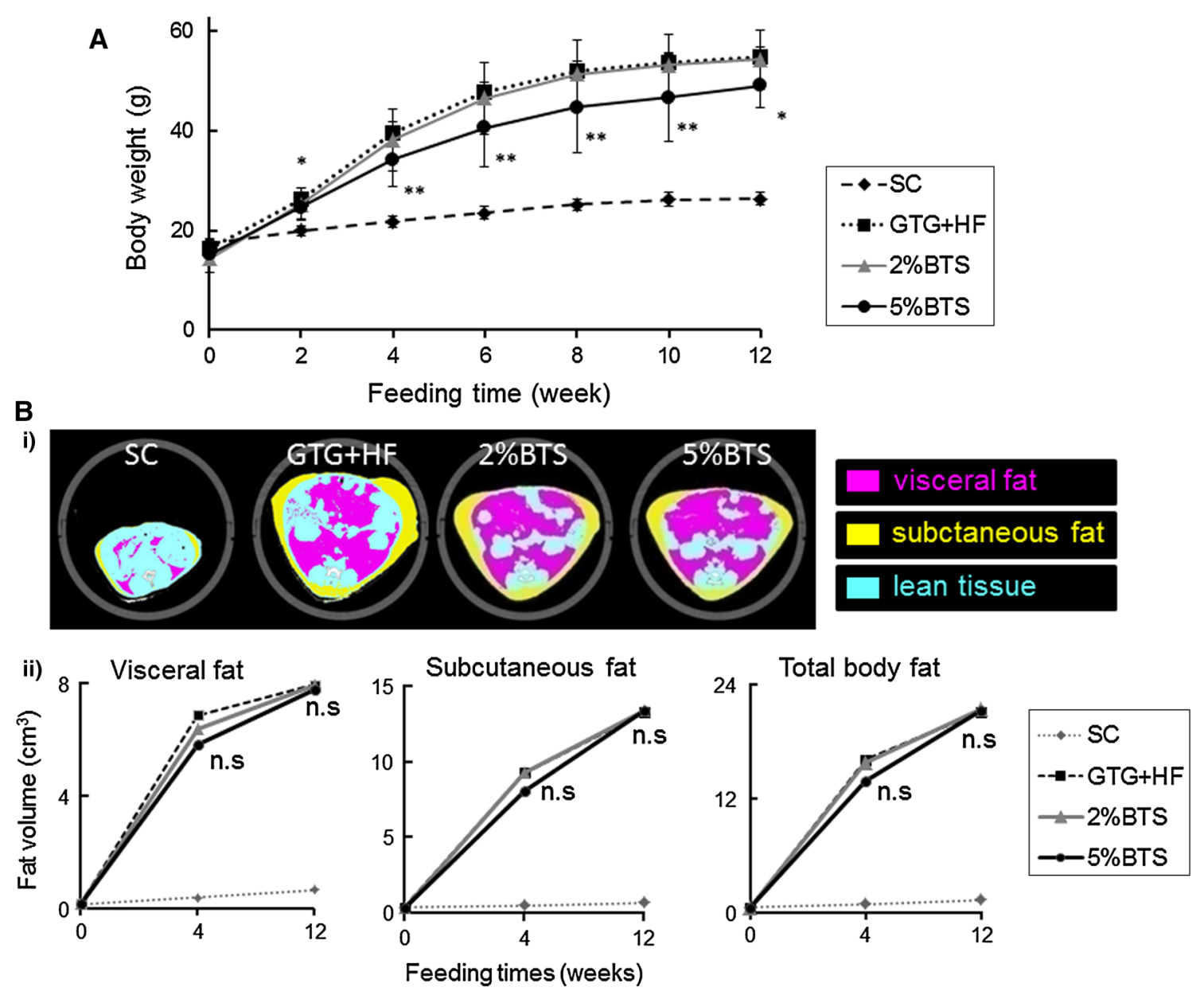

Fig. 1 Effectiveness of BTS for obesity and adiposity. a BTS dosedependently attenuated weight gain in GTG $+\mathrm{HF}$ mice. ${ }^{* *} p<0.01$, ${ }^{*} p<0.05$ vs GTG $+\mathrm{HF}, n=6$. $S C$ standard chow fed mice, $G T G+H F$ GTG treated and HF fed mice, $2 \% B T S$ $2 \% \mathrm{BTS}+\mathrm{GTG}+\mathrm{HF}$ fed mice, $5 \%$ BTS $5 \% \mathrm{BTS}+\mathrm{GTG}+\mathrm{HF}$ fed mice. b Anthropometry and evaluation of subcutaneous fat and visceral fat volumes by abdominal CT $i$ representative

BTS inhibition of hepatic lipid metabolism occurs through induction of adiponectin signaling

We examined plasma adiponectin levels and liver expression of the adiponectin receptors (AdipoR1 and AdipoR2), an important anti-inflammatory cytokine and receptors [18-20], because both plasma adiponectin levels and liver expression of the adiponectin receptors were decreased in $\mathrm{GTG}+\mathrm{HF}$ mice as described previously [9]. Plasma adiponectin level and hepatic expression of AdipoR1, R2 were significantly enhanced by BTS (Fig. 4a). We next investigated the pathways of suppression of hepatic lipid metabolism by adiponectin in the presence of BTS. The expression of SREBP-1c was decreased dose dependently by BTS (Fig. 4b). The phosphorylation of AMPK (P-AMPK/AMPK) was here increased by BTS treatment (Fig. 4c) in parallel with activation of AdipoR1 signaling photomicrographs of abdominal CT scan of SC, GTG + HF, $2 \%$ BTS and $5 \%$ BTS mice shown at 12 weeks. Yellow subcutaneous fat, purple visceral fat, blue lean tissue. ii Time course of increase of volume of subcutaneous and visceral fat. Neither subcutaneous fat nor visceral fat was significantly attenuated by treatment with BTS; $2 \% \mathrm{BTS}, 5 \% \mathrm{BTS}$ vs GTG $+\mathrm{HF}, p=\mathrm{ns}, n=6$

(Fig. 4a). In addition, expression of PPAR- $\gamma$, an activator of AMPK, was increased by BTS treatment (Fig. 4d).

Attenuation of hepatic steatosis with BTS treatment involves activation of fatty acid oxidation

Additionally, the expression of PPAR- $\alpha$ and its target genes, MCAD, involved in mitochondrial $\beta$-oxidation and CYP2E-1 involved in microsomal $\omega$-oxidation [21, 22] were increased by treatment with BTS (Fig. 4e-g).

\section{BTS promotes hepatic lipid export}

We next determined if enhanced secretion of TG from the liver could contribute to the attenuation of hepatic steatosis by BTS treatment. The expression of microsomal triglyceride transfer protein (MTP) known to play a central role in 
Fig. 2 BTS reduces

hepatomegaly and hepatic

steatosis. a Oil red-O staining

and image analysis of livers: Oil

red-O staining showed that BTS

attenuated hepatic steatosis in

GTG + HF fed mice. In

addition, image analysis for Oil

red-O staining of liver sections

confirmed that BTS treatment

attenuated hepatic steatosis.

Plus symbol $p<0.0001$ vs

$2 \% \mathrm{BTS}$, double plus symbol

$p<0.00001$ vs GTG $+\mathrm{HF}$,

$n=6$. b Liver weight and liver/

body weight ratio (liver/body):

the increase of both liver weight and liver/body ratio was significantly attenuated by BTS treatment in a dose dependent manner. Triple asterisk

$p<0.001$, double asterisk

$p<0.01$ vs GTG + HF, plus

symbol $\mathrm{p}<0.05$ vs $2 \%$ BTS,

$n=6$
A
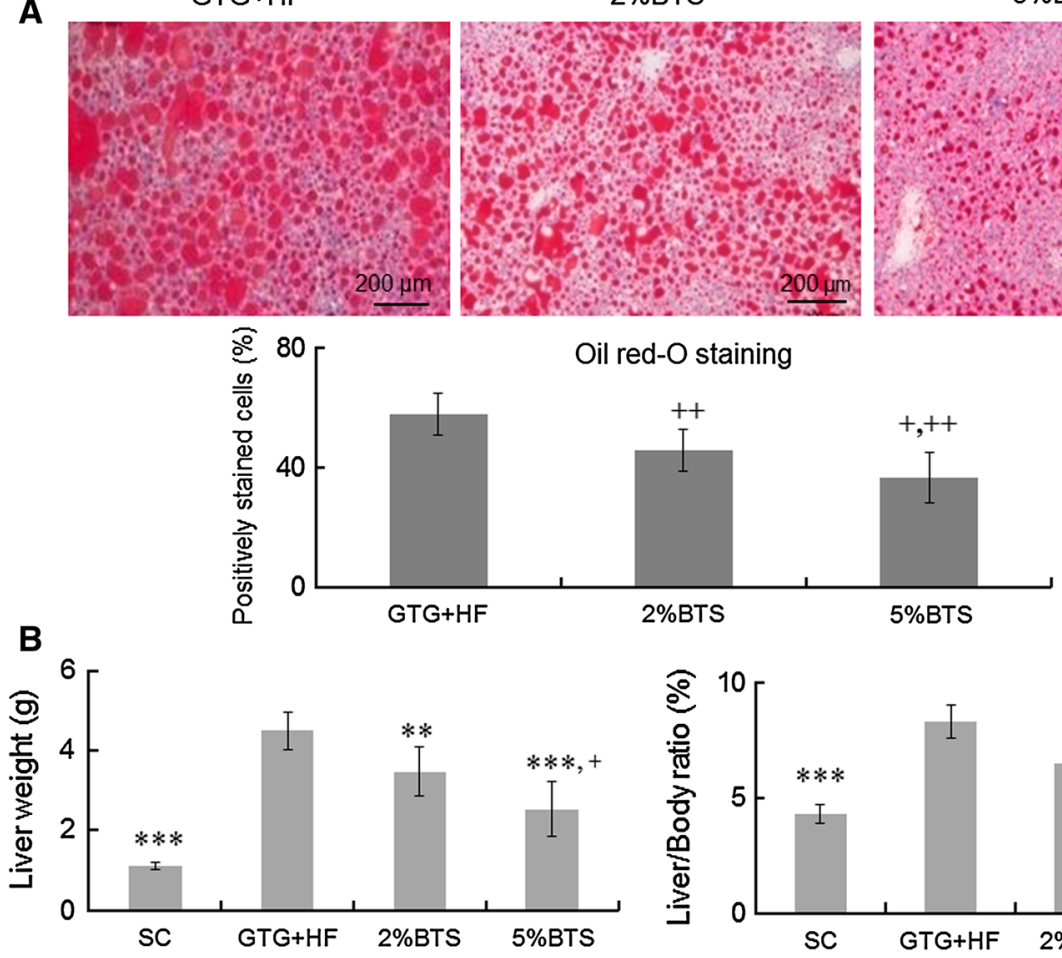

$2 \%$ BTS
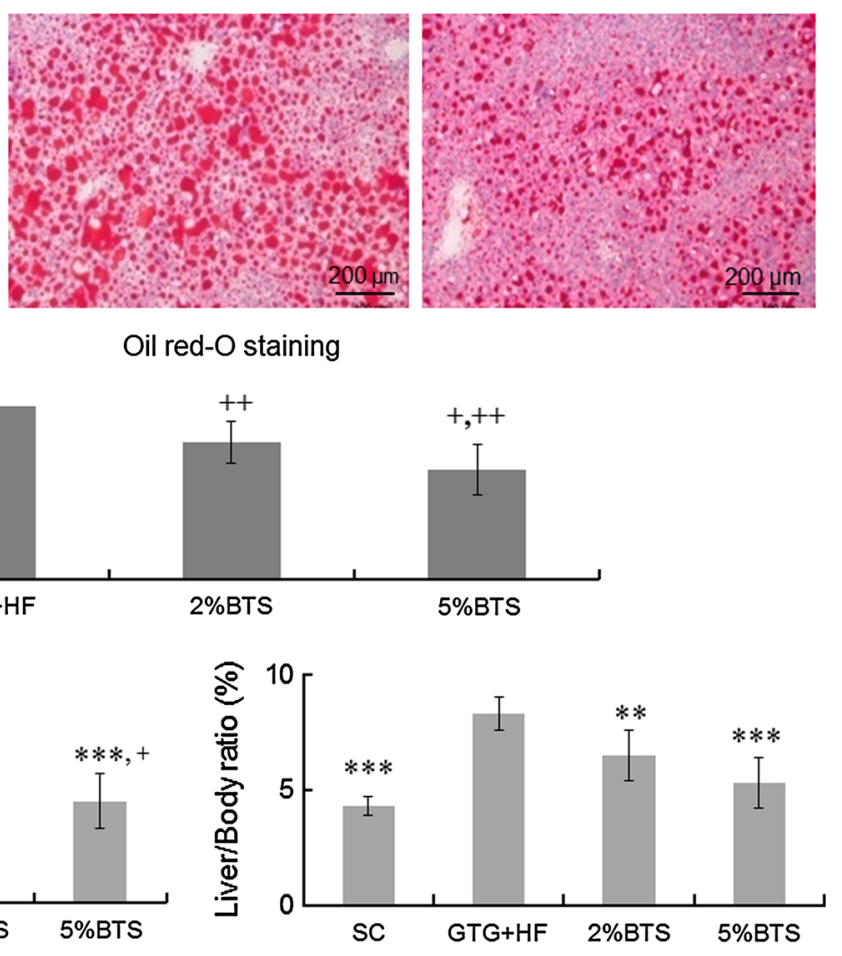

lipoprotein assembly [23] was increased with BTS treatment (Fig. 4h). Interestingly, expression of DGAT2 reported to be involved in the conversion of free fatty acids into TG in the liver [24] was also increased in the BTS treated groups (Fig. 4i).

BTS reduces glucose intolerance and insulin resistance through induction of Akt

Since IR is regarded as a central pathogenic feature of NAFLD [25], we now investigated the effect of BTS treatment on IR. Fasting plasma glucose and insulin levels were markedly reduced in a dose dependent manner by BTS treatment, and QUICKI as an index of IR was also increased by BTS (Fig. 5a). We next evaluated the attenuation of glucose intolerance and IR using the GTT and the ITT in the presence of BTS. GTT revealed that treatment with BTS remarkably attenuated severe glucose intolerance induced by GTG $+\mathrm{HF}$, and ITT showed that treatment with BTS attenuated IR induced by GTG + HF (Fig. 5b). The reduction of IR by BTS involved its promotion of the phosphorylation of Akt (Fig. 5c) an important factor in glucose metabolism [26].

\section{Discussion}

The public health importance of NAFLD [1,2] and the unavailability of proven and effective therapies drive the search for a greater understanding of its pathophysiology and novel therapeutic pathways. In this study, we have clarified the mechanisms through which BTS attenuates NASH based on a novel animal model of NASH [9]. BTS attenuated the GTG + HF induced increases in body and liver weight, serum transaminases, hepatic steatosis, degree of oxidative stress and TNF- $\alpha$ expression (Figs. 1, 2, 3; Table 1) without reducing intake volume of diets (data not shown). However, in contrast to previous reports [6], we did not demonstrate statistically remarkable reduction by BTS, in GTG + HF mice, of induced increases in visceral or subcutaneous fat (Fig. 1b), perhaps because the volume of these fats in our mice were much larger than previously reported [6]. Additionally, the reduction of body weight might mainly be through the reduction of fat accumulation in muscles, since it has been reported that the degree of hepatosteatosis is well correlated with the degree of fat accumulation in muscles [27].

To examine the mechanisms though which BTS attenuated hepatic steatosis, we firstly evaluated the effect of BTS on adiponectin, thought to be a central adipokine in the pathogenesis for NASH $[18,19]$. The expression of AdipoR1 and AdipoR2 in the livers was increased in a dose dependent manner by BTS (Fig. 4a), indicating that BTS could have PPAR- $\alpha$ agonist like effect, since PPAR- $\alpha$ agonists are known to increase expression of AdipoR1 and AdipoR2 [28]. Interestingly, plasma adiponectin levels were also remarkably induced by BTS treatment (Fig. 4a) even though neither visceral nor subcutaneous fat were decreased. These data indicated that BTS through putative 

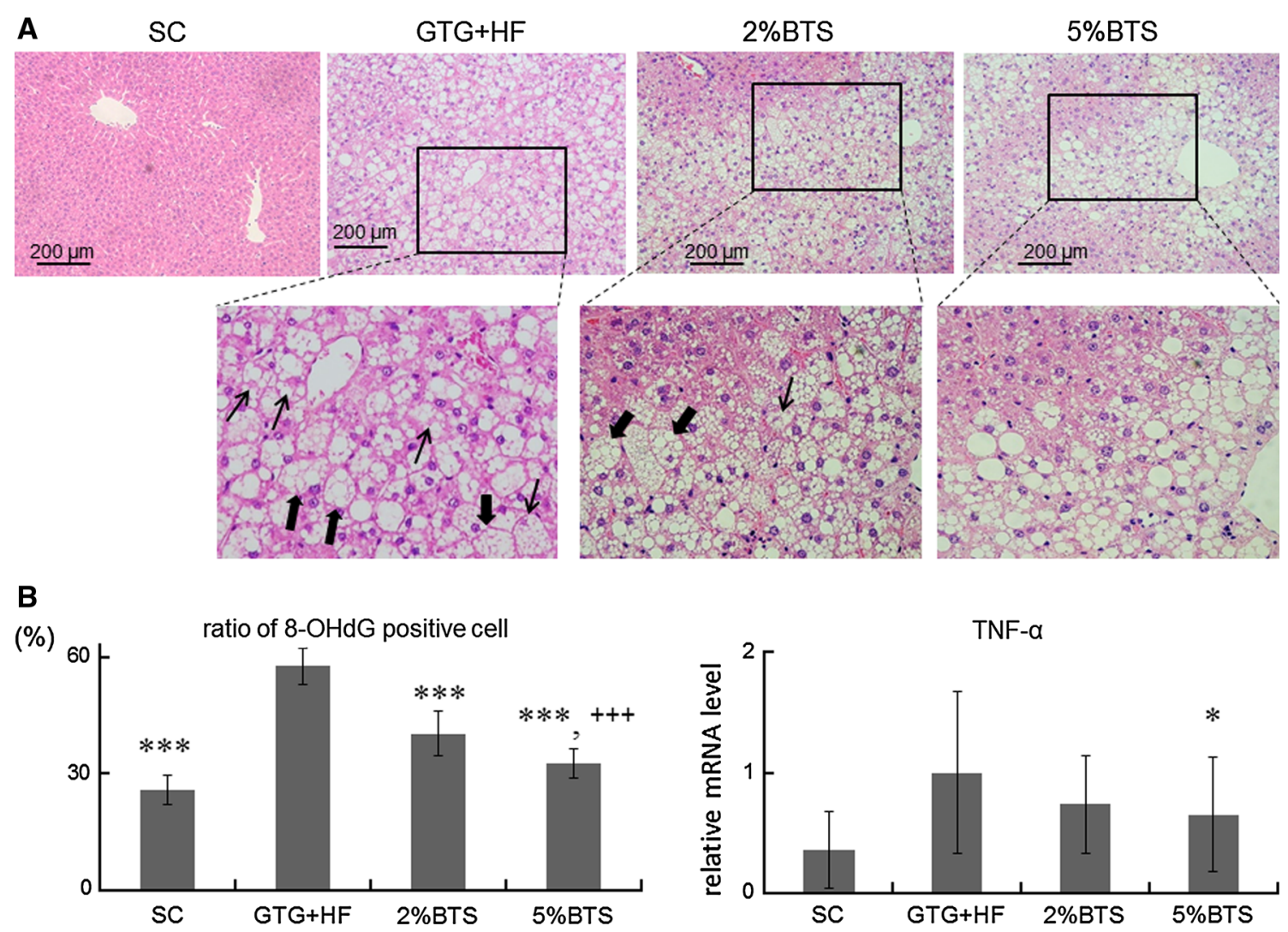

Fig. 3 BTS treatment reduces hepatic inflammation and oxidative stress. a The extent of hepatic inflammation was markedly attenuated with BTS: hepatocyte ballooning and Mallory-Denk bodies in the livers of GTG + HF fed mice were absent in BTS treatment livers. Filled arrow ballooning hepatocyte, arrow Mallory-Denk body. b Oxidative stress and TNF- $\alpha$ expression: BTS reduced hepatic

oxidative stress as shown by reduction of numbers of nuclei stained positive for 8-OHdG in GTG + HF mice. BTS similarly reduced hepatic TNF- $\alpha$ mRNA expression. Triple asterisk $p<0.001$, asterisk $p<0.01$ vs GTG + HF, Triple plus symbol $p<0.001$ vs $2 \% \mathrm{BTS}$, $n=6$

Table 1 Physiological and biochemical analyses in mice treated with BTS

\begin{tabular}{llccc}
\hline & SC $(n=6)$ & GTG + HF $(n=6)$ & $2 \%$ BTS $(n=6)$ & $5 \%$ BTS $(n=6)$ \\
\hline AST (U/L) & $86 \pm 11$ & $310 \pm 114$ & $271 \pm 27$ & $170 \pm 46^{*}$ \\
ALT (U/L) & $31 \pm 9$ & $514 \pm 170$ & $433 \pm 37$ & $299 \pm 133 *$ \\
TG $(\mathrm{mg} / \mathrm{dL})$ & $36 \pm 7$ & $37 \pm 13$ & $52 \pm 11^{*}$ & $49 \pm 8$ \\
\hline
\end{tabular}

Serum ALT and AST levels were significantly reduced in mice treated with GTG $+\mathrm{HF}+5 \%$ BTS compared to the control GTG $+\mathrm{HF}$ group, $* p<0.05$. There was no change in serum TG level between the GTG + HF $+5 \%$ BTS and GTG + HF groups although TG was elevated by $2 \%$ BTS compared to GTG + HF $(* p<0.05)$

$* p<0.05$ vs GTG + HF, $n=6$

PPAR- $\gamma$ effects may also function to increase serum adiponectin, since PPAR- $\gamma$ agonists have been shown to positively regulate serum adiponectin independently of adipose tissue volume regulation $[28,29]$.

To now study the mechanisms of inhibition of liver lipid metabolism by BTS in the presence of the activated adiponectin signaling pathway, AdipoR1 signaling pathways and their target genes were examined. It is known that activated AdipoR1 signaling decreases expression of SREBP-1c [30], a key regulator of hepatic fatty acid synthesis [31, 32], through AMPK activation [33]. Here, we showed increased phosphorylation of AMPK by BTS in parallel with activated AdipoR1 signaling (Fig. 4c). Additionally, the phosphorylation of AMPK could also have been induced by activated PPAR- $\gamma$, in BTS treated mice, since as above BTS could also have PPAR- $\gamma$ agonist like actions. Moreover, in the livers of mice treated with BTS, there was a reduced expression of SREBP-1c (Fig. 4b) in parallel with activated adiponectin signaling and phosphorylation of AMPK. 
A

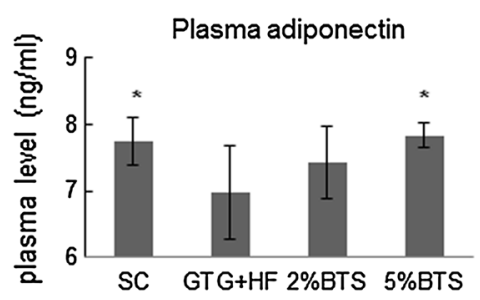

B

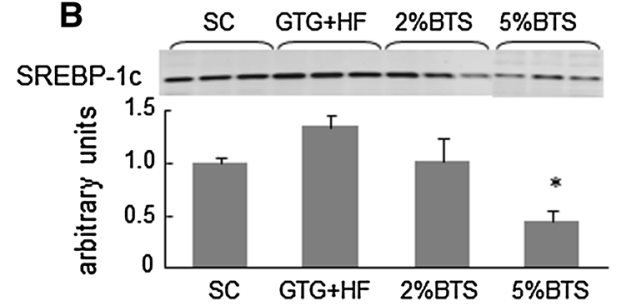

E

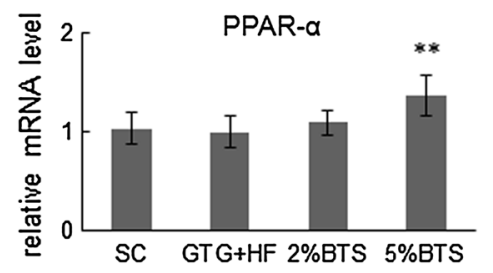

H

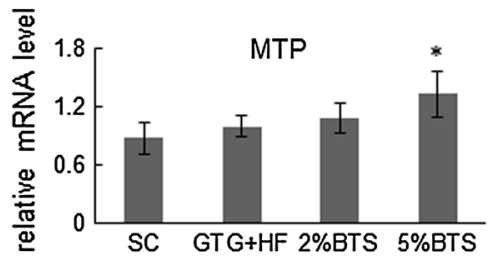

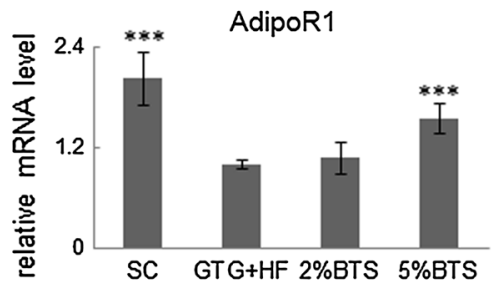

C

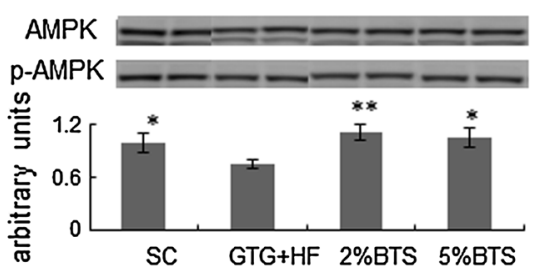

$\mathbf{F}$

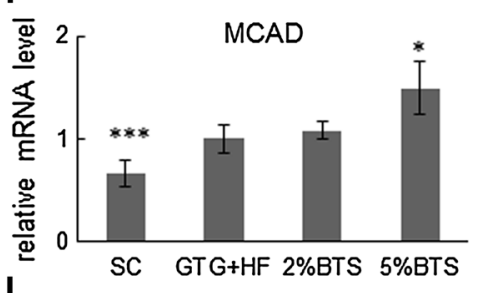
I

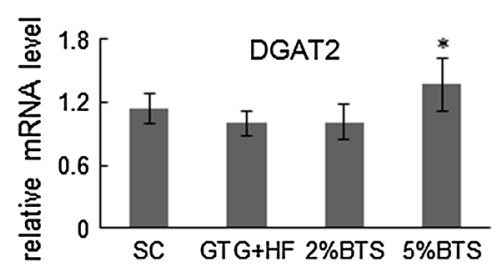

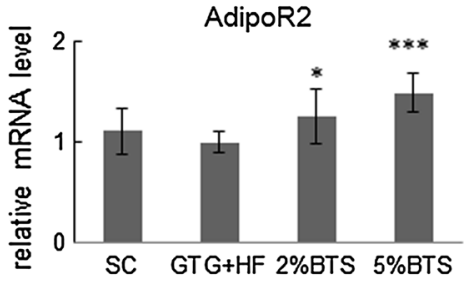

D

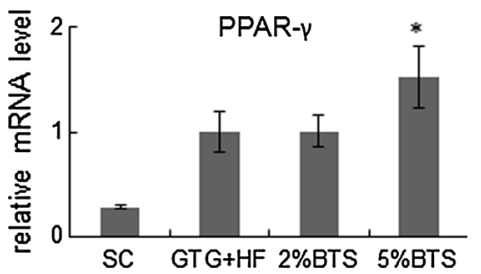

G

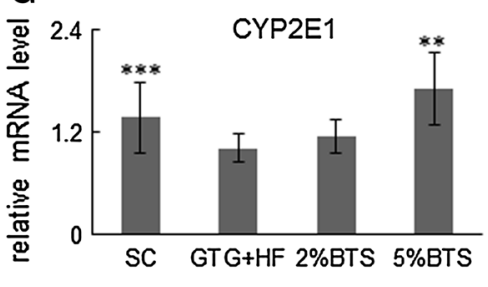

Fig. 4 BTS attenuates hepatosteatosis through activation of adiponectin. a Plasma adiponectin and expression of adiponectin receptors 1 (AdipoR1) and 2 (AdipoR2): plasma adiponectin levels and hepatic mRNA expression of AdipoR1 and AdipoR2 were dose dependently increased by BTS. Asterisk $p<0.05$, triple asterisk $p<0.001$ vs GTG $+\mathrm{HF}, n=6$. b-d AdioR1 signaling and target genes expression: BTS induced AdipoR1 signaling suppressed nuclear expression of SREBP-1c $(n=4)$ through phosphorylated AMPK $(n=4)$. Expression of PPAR- $\gamma$ mRNA was also increased by BTS treatment

TNF- $\alpha$ expression is also known to regulate expression of SREBP-1c through activation of AMPK [31, 34]. Therefore, the suppression of TNF- $\alpha$ expression by BTS, as observed here, could also have contributed to the decreased SREBP1c expression. Taken together, therefore, the data here suggest that activation of AdipoR1 signaling and consequent suppression of SREBP-1c may be central mechanisms through which BTS attenuates hepatic steatosis.

BTS may also have enhanced hepatic fatty acid oxidation via AdipoR2 signaling, known to increase the expression of PPAR- $\alpha$ [24] and its fatty acid oxidation related target genes. Here, the expression of PPAR- $\alpha$ (Fig. 4e) and its target gene MCAD (Fig. 4f) and CYP2E1 (Fig. 4g), were increased by treatment with BTS. Therefore, activated fatty $(n=6)$. Asterisk $p<0.05$, double asterisk $p<0.01$ vs GTG + HF. e-g Expression of PPAR- $\alpha$ and its target genes: Expression of PPAR$\alpha$ mRNA was increase by BTS $(n=6)$. Expression of MCAD $(n=6)$ and CYP2E1 $(n=6)$, PPAR- $\alpha$ target genes, was similarly increased by BTS. Asterisk $p<0.05$, double asterisk $p<0.01$, triple asterisk $p<0.001$ vs GTG + HF. h, i BTS promotes hepatic lipid export: MRNA expression of MTP $(n=6)$ and DGAT2 $(n=6)$ was increased by treatment with BTS. Asterisk $p<0.05$ vs GTG + HF

acids oxidation could also contribute to the attenuation of hepatic steatosis by BTS. Activation of fatty acid oxidation would be expected to increase production of reactive oxygen species (ROS) in the liver [35, 36]. BTS treatment here reduced ROS levels, probably contributing to the improved hepatic inflammation observed with BTS.

Additionally, MTP expression was confirmed here to be increased by treatment with BTS, possibly contributing to attenuation of steatosis by increasing hepatic export of TG (Fig. 4h). Interestingly, expression of DGAT2 was also increased by BTS treatment (Fig. 4i). Reduced hepatic accumulation of TG in BTS-treated livers may therefore have been due to increased MTP expression and increased expression of DGAT2 to reduce the content of FFA. 

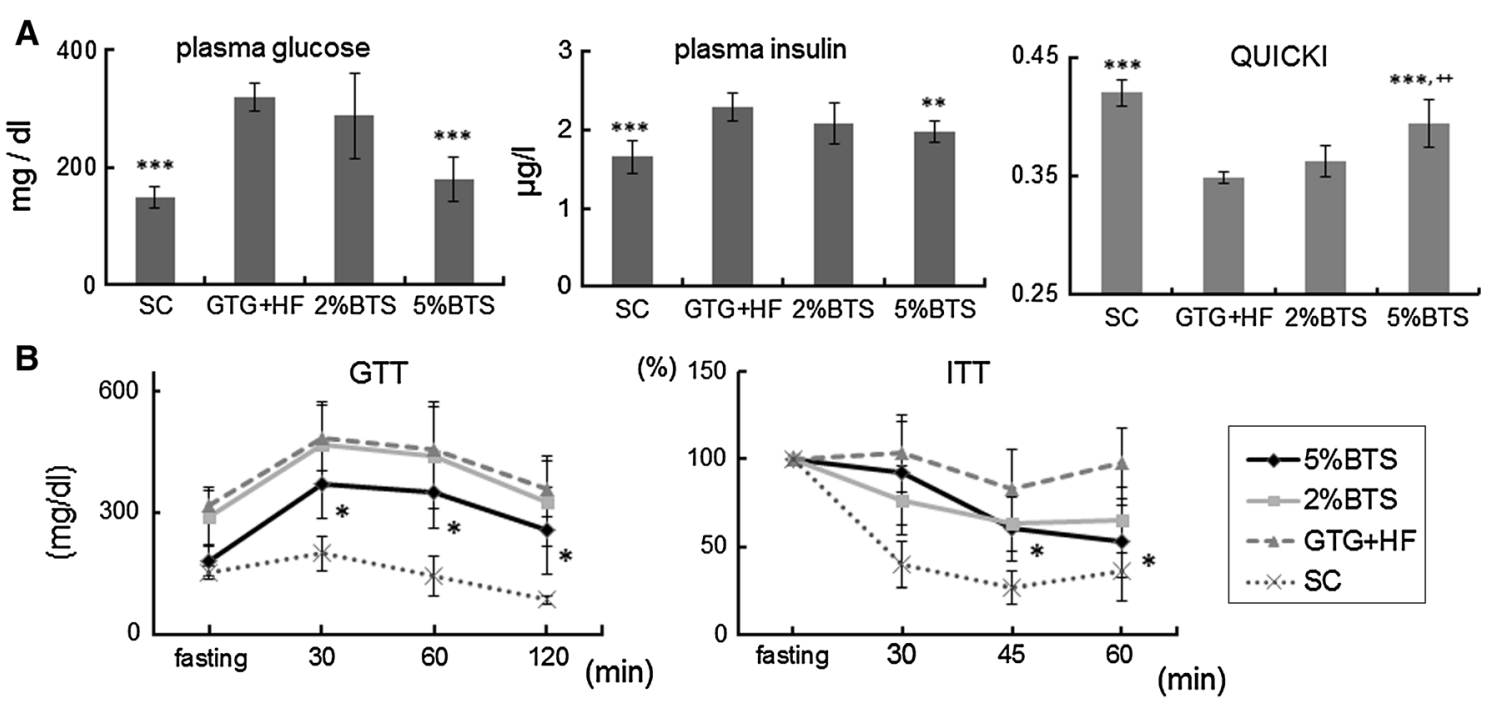

(\%) 150 ITT
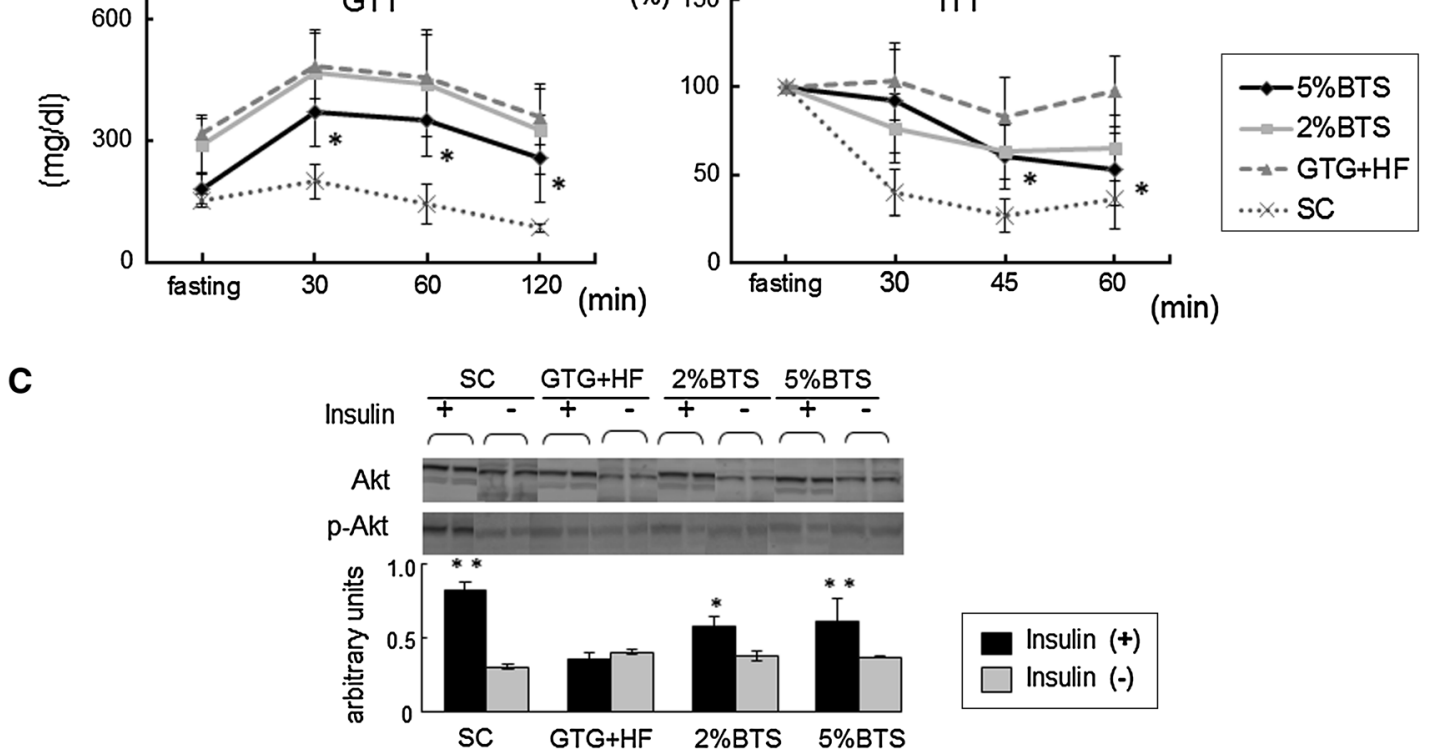

Fig. 5 Glucose intolerance and insulin resistance (IR) were attenuated by BTS. a Fasting plasma glucose, insulin levels and QUICKI: increased level of fasted plasma insulin and glucose were decreased, and QUICKI increased by treatment with $5 \%$ BTS, and ITT confirmed severe IR double asterisk $p<0.01$, triple asterisk $p<0.001$ vs GTG $+\mathrm{HF}$, double plus symbol $p<0.01$ vs $2 \% \mathrm{BTS}$, $n=6$. b Glucose tolerance test (GTT) and insulin tolerance test

Furthermore, increased expression of DGAT2 might also have contributed to the reduction of ROS production by reducing hepatic FFA availability.

We next also investigated the effect of BTS on IR. GTG + HF induced elevation of fasting plasma glucose and plasma insulin were markedly reduced by BTS treatment, whilst QUICKI was markedly increased by BTS (Fig. 5a). In addition, ITT also was attenuated by BTS treatment. Furthermore, GTT showed that BTS remarkably attenuated severe glucose intolerance induced by GTG + HF (Fig. 5b). Phosphorylation of hepatic Akt after administration of insulin was reduced by treatment of BTS (Fig. 5c). The mechanisms through which BTS improves insulin sensitivity may involve Akt phosphorylation triggered through as yet uncertain mechanisms, but possibly involving induction of adiponectin signaling by BTS, since adiponectin is known to be involved in the suppression of hepatic gluconeogenesis and insulin secretion by activating AMPK [37].

In conclusion, therefore, BTS-a Japanese anti-obesity herbal (Kampo) medicine-is an effective preventive agent
(ITT): GTT revealed severe glucose intolerance was attenuated, and ITT confirmed IR was attenuated by $5 \%$ BTS. Asterisk $p<0.05$ vs GTG $+\mathrm{HF}, n=6$. c Involvement of Akt in BTS reduction of IR: phosphorylation of Akt was involved in the reduction of IR by BTS treatment as reflected by increased phosphorylation of Akt in the livers of BTS treated mice upon insulin administration. Asterisk $p<0.05$, double asterisk $p<0.01 \mathrm{vs} \mathrm{GTG}+\mathrm{HF}, n=4$

against the development of NASH. The challenge is now to identify the active components of BTS to allow its refinement and the rational design of more potent analogues.

Acknowledgments Grants-in-Aid for Scientific Research (C) 2009 Grant \# 20590785, The Ministry of Education, Science, Sports and Culture, Japan (Masafumi Ono). Wellcome Trust, UK (Jude A. Oben).

Conflict of interest The authors declare that they have no conflict of interest.

Open Access This article is distributed under the terms of the Creative Commons Attribution Noncommercial License which permits any noncommercial use, distribution, and reproduction in any medium, provided the original author(s) and the source are credited.

\section{References}

1. Ioannou GN, Boyko EJ, Lee SP. The prevalence and predictors of elevated serum aminotransferase activity in the United States in 1999-2002. Am J Gastroenterol. 2006;101:76-82. 
2. James O, Day C. Non-alcoholic steatohepatitis: another disease of affluence. Lancet. 1999;353:1634-6.

3. Guha IN, Parkes J, Roderick P, Chattopadhyay D, Cross R, Harris $\mathrm{S}$, et al. Noninvasive markers of fibrosis in nonalcoholic fatty liver disease: validating the European liver fibrosis panel and exploring simple markers. Hepatology. 2008;47:455-60.

4. Hioki C, Yoshimoto K, Yoshida T. Efficacy of bofu-tsusho-san, an oriental herbal medicine, in obese Japanese women with impaired glucose tolerance. Clin Exp Pharmacol Physiol. 2004; 31:614-9.

5. Yamakawa J, Ishigaki Y, Takano F, Takahashi T, Yoshida J, Moriya J, et al. The Kampo medicines Orengedokuto, Bofutsushosan and Boiogito have different activities to regulate gene expressions in differentiated rat white adipocytes: comprehensive analysis of genetic profiles. Biol Pharm Bull. 2008;31:2083-9.

6. Shimada T, Kudo T, Akase T, Aburada M. Preventive effects of Bofutsushosan on obesity and various metabolic disorders. Biol Pharm Bull. 2008;31:1362-7.

7. Nakayama T, Suzuki S, Kudo H, Sassa S, Nomura M, Sakamoto S. Effects of three Chinese herbal medicines on plasma and liver lipids in mice fed a high-fat diet. J Ethnopharmacol. 2007;109: 236-40.

8. Sakamoto S, Takeshita S, Sassa S, Zuki S, Ishikawa Y, Kudo H. Effects of colestimide and/or Bofu-tsusho-san on plasma and liver lipids in mice fed a high-fat diet. In vivo. 2005;19:1029-33.

9. Ogasawara M, Hirose A, Ono M, Aritake K, Nozaki Y, Takahashi $\mathrm{M}$, et al. A novel and comprehensive mouse model of human non-alcoholic steatohepatitis with the full range of dysmetabolic and histological abnormalities induced by gold thioglucose and a high-fat diet. Liver Int. 2011;31:542-51.

10. Oike Y, Akao M, Yasunaga K, Yamauchi T, Morisada T, Ito Y, et al. Angiopoietin-related growth factor antagonizes obesity and insulin resistance. Nat Med. 2005;11:400-8.

11. Fan W, Yanase T, Nomura M, Okabe T, Goto K, Sato T, et al. Androgen receptor null male mice develop late-onset obesity caused by decreased energy expenditure and lipolytic activity but show normal insulin sensitivity with high adiponectin secretion. Diabetes. 2005;54:1000-8.

12. Hirose A, Ono M, Saibara T, Nozaki Y, Masuda K, Yoshioka A, et al. Angiotensin II type 1 receptor blocker inhibits fibrosis in rat nonalcoholic steatohepatitis. Hepatology. 2007;45:1375-81.

13. Albrecht C, Knaapen AM, Becker A, Höhr D, Haberzettl P, van Schooten FJ, et al. The crucial role of particle surface reactivity in respirable quartz-induced reactive oxygen/nitrogen species formation and APE/Ref-1 induction in rat lung. Respir Res. 2005;6:129.

14. Guerre-Millo M, Rouault C, Poulain P, André J, Poitout V, Peters JM, et al. PPAR-alpha-null mice are protected from high-fat dietinduced insulin resistance. Diabetes. 2001;50:2809-14.

15. Ma K, Cabrero A, Saha PK, Kojima H, Li L, Chang BH, et al. Increased beta-oxidation but no insulin resistance or glucose intolerance in mice lacking adiponectin. J Biol Chem. 2002;277: 34658-61.

16. Taniguchi CM, Ueki K, Kahn R. Complementary roles of IRS-1 and IRS-2 in the hepatic regulation of metabolism. J Clin Invest. 2005;115:718-27.

17. DeBose-Boyd RA, Ou J, Goldstein JL, Brown MS. Expression of sterol regulatory element-binding protein 1c (SREBP-1c) mRNA in rat hepatoma cells requires endogenous LXR ligands. Proc Natl Acad Sci USA. 2001;98:1477-82.

18. Hui JM, Hodge A, Farrell GC, Kench JG, Kriketos A, George J. Beyond insulin resistance in NASH: TNF-alpha or adiponectin? Hepatology. 2004;40:46-54.

19. Kamada Y, Takehara T, Hayashi N. Adipocytokines and liver disease. J Gastroenterol. 2008;43:811-22.
20. Tomita K, Oike Y, Teratani T, Taguchi T, Noguchi M, Suzuki T, et al. Hepatic AdipoR2 signaling plays a protective role against progression of nonalcoholic steatohepatitis in mice. Hepatology. 2008;48:458-73.

21. Rao MS, Reddy JK. PPARalpha in the pathogenesis of fatty liver disease. Hepatology. 2004;40:783-6.

22. Reddy JK, Hashimoto T. Peroxisomal beta-oxidation and peroxisome proliferator-activated receptor alpha: an adaptive metabolic system. Annu Rev Nutr. 2001;21:193-230.

23. Namikawa C, Shu-Ping Z, Vyselaar JR, Nozaki Y, Nemoto Y, Ono M, et al. Polymorphisms of microsomal triglyceride transfer protein gene and manganese superoxide dismutase gene in nonalcoholic steatohepatitis. J Hepatol. 2004;40:781-6.

24. Yamaguchi K, Yang L, McCall S, Huang J, Yu XX, Pandey SK, et al. Inhibiting triglyceride synthesis improves hepatic steatosis but exacerbates liver damage and fibrosis in obese mice with nonalcoholic steatohepatitis. Hepatology. 2007;45:1366-74.

25. Postic C, Girard J. Contribution of de novo fatty acid synthesis to hepatic steatosis and insulin resistance: lessons from genetically engineered mice. J Clin Invest. 2008;118:829-38.

26. Whiteman EL, Cho H, Birnbaum MJ. Role of Akt/protein kinase B in metabolism. Trends Endocrinol Metab (TEM). 2002;13:444-51.

27. Kitajima Y, Eguchi Y, Ishibashi E, Nakashita S, Aoki S, Toda S, et al. Age-related fat deposition in multifidus muscle could be a marker for nonalcoholic fatty liver disease. J Gastroenterol. 2010; 45:218-24.

28. Tsuchida A, Yamauchi T, Takekawa S, Hada Y, Ito Y, Maki T, et al. Peroxisome proliferator-activated receptor (PPAR)alpha activation increases adiponectin receptors and reduces obesityrelated inflammation in adipose tissue: comparison of activation of PPARalpha, PPARgamma, and their combination. Diabetes. 2005;54:3358-70.

29. Waki H, Yamauchi T, Kamon J, Ito Y, Uchida S, Kita S, et al. Impaired multimerization of human adiponectin mutants associated with diabetes. Molecular structure and multimer formation of adiponectin. J Biol Chem. 2003;278:40352-63.

30. Shklyaev S, Aslanidi G, Tennant M, Prima V, Kohlbrenner E, Kroutov V, et al. Sustained peripheral expression of transgene adiponectin offsets the development of diet-induced obesity in rats. Proc Natl Acad Sci USA. 2003;100:14217-22.

31. Browning JD, Horton JD. Molecular mediators of hepatic steatosis and liver injury. J Clin Invest. 2004;114:147-52.

32. Yamauchi T, Nio Y, Maki T, Kobayashi M, Takazawa T, Iwabu $\mathrm{M}$, et al. Targeted disruption of AdipoR1 and AdipoR2 causes abrogation of adiponectin binding and metabolic actions. Nat Med. 2007;13:332-9.

33. Yano W, Kubota N, Itoh S, Kubota T, Awazawa M, Moroi M, et al. Molecular mechanism of moderate insulin resistance in adiponectin-knockout mice. Endocr J. 2008;55:515-22.

34. Endo M, Masaki T, Seike M, Yoshimatsu H. TNF-alpha induces hepatic steatosis in mice by enhancing gene expression of sterol regulatory element binding protein-1c (SREBP-1c). Exp Biol Med (Maywood). 2007;232:614-21.

35. Evans JL, Goldfine ID, Maddux BA, Grodsky GM. Are oxidative stress-activated signaling pathways mediators of insulin resistance and beta-cell dysfunction? Diabetes. 2003;52:1-8.

36. Leclercq IA, Farrell GC, Field J, Bell DR, Gonzalez FJ, Robertson GR. CYP2E1 and CYP4A as microsomal catalysts of lipid peroxides in murine nonalcoholic steatohepatitis. J Clin Invest. 2000;105:1067-75.

37. Yamauchi T, Kamon J, Minokoshi Y, Ito Y, Waki H, Uchida S, et al. Adiponectin stimulates glucose utilization and fatty-acid oxidation by activating AMP-activated protein kinase. Nat Med. 2002;8:1288-95. 